\title{
MicroRNA-301a-3p overexpression promotes cell invasion and proliferation by targeting runt-related transcription factor 3 in prostate cancer
}

\author{
LI FAN, YAO WANG, WEI HUO and WEI-HUA WANG \\ Department of Urology, China-Japan Union Hospital of Jilin University, Changchun, Jilin 130033, P.R. China
}

Received December 10, 2016; Accepted April 6, 2018

DOI: $10.3892 / \mathrm{mmr} .2019 .10650$

\begin{abstract}
MicroRNAs (miRNAs) are known to serve a role in tumorigenic programs. The dysregulated expression of miR-301a-3p may affect the progression of various types of human cancer; however, the expression and the role of miR-301a-3p in prostate cancer are still unclear. The present study aimed to clarify the role and molecular mechanism of miR-301a-3p in prostate cancer. The results demonstrated that the expression of miR-301a-3p was significantly upregulated in human prostate cancer tissues and in several prostate cancer cell lines. In vitro overexpression of miR-301a-3p notably increased prostate cancer cell proliferation and invasion. Bioinformatics analysis revealed that runt-related transcription factor 3 (RUNX3) may be a target of miR-301a-3p, which was confirmed by Dual-luciferase reporter assay. Western blot analysis also demonstrated that miR-301a-3p regulated the protein expression levels of RUNX3. In addition, the results indicated that miR-301a-3p may regulate the Wnt signaling pathway, and rescue experiments indicated that RUNX3 contributed to the effects of miR-301a-3p on cell proliferation and invasion through Wnt signaling. In conclusion, these findings suggested that miR-301a-3p may promote prostate cancer cell invasion and proliferation by targeting RUNX3, and provided insight into understanding prostate cancer pathogenesis. miR-301a-3p may be a potential therapeutic candidate to treat prostate cancer.
\end{abstract}

\section{Introduction}

Prostate cancer is one of the most commonly diagnosed cancers in men, resulting in significant mortality and morbidity (1). A

Correspondence to: Dr Wei-Hua Wang, Department of Urology, China-Japan Union Hospital of Jilin University, 126 Xiantai Street, Changchun, Jilin 130033, P.R. China

E-mail: wangweihuajilin@163.com

Abbreviations: miRNA/miR, microRNA; RUNX3, runt-related transcription factor 3; UTR, untranslated region

Key words: microRNA-301a-3p, runt-related transcription factor 3, prostate cancer, Wnt signaling pathway previous study reported that a number of signaling pathways, including androgen receptor, phosphoinositide 3-kinase, mitogen-activated protein kinase and Wnt, may be involved in the progression of prostate cancer (2); however, the molecular mechanisms that control this progression requires further study. MicroRNAs (miRNAs) are small, non-coding RNAs that target the 3'-untranslated region (UTR) of target mRNA transcripts to suppress translation or to induce mRNA degradation (3). miRNAs serve crucial roles in various cellular processes, including proliferation, apoptosis, differentiation, migration and invasion (4). Previous studies have reported that dysregulated miRNA expression may be a factor in various types of cancer (5). A previous study has also suggested that miRNAs may serve a number of roles in prostate cancer pathogenesis (6); however, their precise roles in the pathogenesis and the possible mechanisms remain unclear.

miRNAs function by regulating the expression of target genes. Runt-related transcription factor 3 (RUNX3) was previously predicted to be a target gene of miR-301a-3p (7). Downregulated RUNX3 expression has been implicated in gastric cancer, lung adenocarcinoma and hepatocellular carcinoma (7-9), and was associated with increased chemotherapy resistance (10).

A previous study reported that miR-301a-3p was overexpressed in hepatocellular carcinoma, pancreatic tumor tissues and small cell lung cancer compared with adjacent benign tissues (11); however, the role of miR-301a-3p in prostate cancer remains unknown. The present study aimed to investigate the expression levels and biological roles of miR-301a-3p in prostate cancer progression. The data demonstrated that miR-301a-3p expression levels were significantly upregulated in human prostate cancer tissues and cell lines and suggested that overexpression of miR-301a-3p may promote prostate cancer cell proliferation and invasion. RUNX3 was verified as a direct target of miR-301a-3p. Therefore, miR-301a-3p may promoted prostate cancer cell proliferation and invasion by targeting RUNX3.

\section{Materials and methods}

Clinical specimens. Paired prostate malignant cancer tissues and adjacent normal prostate tissues $(n=15)$ were obtained from patients with prostate cancer who underwent resection surgery at the China-Japan Union Hospital of Jilin University (Changchun, China). Written informed consent was obtained from the participating patients prior to the start of the study, 
and the study was approved by The Institutional Human Experiment and Ethics Committee of China-Japan Union Hospital of Jilin University.

Cell lines and cell culture. Human prostate cancer cell lines (PC-3, LNCaP and DU-145), normal prostate epithelial cells (RWPE-1) and 293T cells were purchased from the American Type Culture Collection (Manassas, VA, USA). Prostate cancer cell lines and 293T cells were grown in RPMI-1640 and Dulbecco's modified Eagle's medium (Gibco; Thermo Fisher Scientific, Inc., Waltham, MA, USA), respectively, both supplemented with $10 \%$ fetal bovine serum (FBS, Gibco, Thermo Fisher Scientific, Inc.), $100 \mathrm{mg} / \mathrm{ml}$ streptomycin and $100 \mathrm{U} / \mathrm{ml}$ penicillin (Gibco; Thermo Fisher Scientific, Inc.). RWPE-1 cells were grown in keratinocyte serum-free media supplemented with $5 \mathrm{ng} / \mathrm{ml}$ human recombinant epidermal growth factor, $0.05 \mathrm{mg} / \mathrm{ml}$ bovine pituitary extract, $100 \mathrm{mg} / \mathrm{ml}$ streptomycin and $100 \mathrm{U} / \mathrm{ml}$ penicillin (Gibco; Thermo Fisher Scientific, Inc.). All cells were cultured in a humidified incubator containing $5 \% \mathrm{CO}_{2}$ at $37^{\circ} \mathrm{C}$.

$R N A$ isolation and reverse transcription-quantitative polymerase chain reaction ( $R T-q P C R)$. TRIzol reagent (Invitrogen; Thermo Fisher Scientific, Inc.) was used to extract total RNA from $6 \mathrm{~mm}$ tissue sample or $1 \times 10^{7}$ cell from PC-3, LNCaP and DU-145 cell lines respectively, according to the manufacturer's protocol. The primers were synthesized by the Shanghai Sangon Biological Engineering and Technology Service (Shanghai, China). For gene expression detection, cDNA was synthesised from total RNA, RT-qPCR mixture system contained the cDNA, primers and SYBR-Green qPCR Master Mix. The thermocycling conditions for RT-qPCR were: $94^{\circ} \mathrm{C} 5 \mathrm{~min}$, followed by 45 cycles of $94^{\circ} \mathrm{C}$ for $45 \mathrm{sec}, 59^{\circ} \mathrm{C}$ for $45 \mathrm{sec}$ and $72^{\circ} \mathrm{C}$ for $1 \mathrm{~min}$. miRNA expression levels were assessed by the TaqMan stem-loop RT-PCR method according to the manufacturer's protocol (Applied Biosystems; Thermo Fisher Scientific, Inc.). Reverse transcription was performed using the one-step PrimerScript miRNA cDNA synthesis kit to synthesize cDNA from total RNA (Takara Biotechnology Co., Ltd., Dalian, China). In brief, $10 \mu \mathrm{l}$ qPCR mix, $0.4 \mu \mathrm{l}$ forward primer, $0.4 \mu \mathrm{l}$ reverse primer, $2 \mu \mathrm{l} \mathrm{cDNA}$ and $\mathrm{dd}_{2} \mathrm{O}$ were mixed. The thermocycling conditions for RT-qPCR were $95^{\circ} \mathrm{C}$ for $5 \mathrm{~min}$, followed by 30 cycles of $95^{\circ} \mathrm{C}$ for $5 \mathrm{sec}$, $60^{\circ} \mathrm{C}$ for $10 \mathrm{sec}$ and $72^{\circ} \mathrm{C}$ for $1 \mathrm{~min}$. The U6 small nuclear RNA (RNU6B) was used for miRNA expression normalization. mRNA expression level of RUNX3 was normalized to $\beta$-actin. Relative gene or miRNA expression was quantified by $2^{-\triangle \Delta C q}$ method (12). Primers were as follows: RUNX3, forward 5'-GCTGTTATGCGTATTCCCGTAG-3' and reverse, 5'-TGA AGTGGCTTGTGGTGCTGAGTGA-3'; cyclin D1 forwards, 5'-AACTACCTGGACCGCTTCCT-3' and reverse, 5'-CCA CTTGAGCTTGTTCACCA-3'; c-myc, forward, 5'-TCTTAG TCTTTTTCTTAATAGGG-3' and reverse 5'-GGTATCTGG ACCTCACTGACAAG-3'; $\beta$-actin, forward 5'-TTAATAGTC ATTCCAAATATGA-3' and reverse, 5'-GGGACAAAAAAG GGGGAAGG-3'; miR-301a-3p, forward 5'-CGTGCGAAG CTCAGGAGGG-3' and reverse, 5'-TGGCTGTCGTGGACT GCG-3'; RNU6B, 5'-GGACATCCGATAAAATTGGAA CGATACAG-3' and reverse, 5'-AATTTGGACCATTTCTCG
ATTTATGCGTGT-3'. The experiment was repeated three times.

Celltransfection.miR-301a-3pmimics(cat.no.4464066),antisense nucleotides of miR-301a-3p (anti- miR-301a-3p; cat.no. AM17000) and control oligonucleotides (miR-NC; cat. no. 4464058) were purchased from Thermo Fisher Scientific, Inc. Prostate cancer cells (LNCaP and DU-145) at $1 \times 10^{8}$ were transiently transfected with $50 \mathrm{nM}$ miRNA [as determined by dose response analysis (data not shown)] using Lipofectamine ${ }^{\circledR} 2000$ (Thermo Fisher Scientific, Inc.), according to the manufacturer's instructions. Normal prostate cancer cells were used as a control. Following $48 \mathrm{~h}$ incubation at $37^{\circ} \mathrm{C}$, transfection efficiency was detected by RT-qPCR analysis, as aforementioned.

Cell proliferation assay. Cell proliferation was measured by MTT assay. LNCaP and DU-145 cells ( $1 \times 10^{4}$ cells/well) were plated in 96-well plates and cultured for $24 \mathrm{~h}$ at $37^{\circ} \mathrm{C}$, respectively. The cells were transfected with $50 \mathrm{nM}$ miR-301a-3p mimics or anti-miR-301a-3p for $1,3,5$ and 7 days at $37^{\circ} \mathrm{C}$. An untransfected control was also used. Following each transfection period, $20 \mu 1$ MTT solution $(5 \mathrm{mg} / \mathrm{ml}$; Sigma-Aldrich; Merck KGaA, Darmstadt, Germany) was added to each well and incubated at $37^{\circ} \mathrm{C}$ for $4 \mathrm{~h}$. The medium was discarded, and the formazan crystals were dissolved by adding $200 \mu \mathrm{l}$ dimethyl sulfoxide per well. The absorbance was measured at a wavelength of $490 \mathrm{~nm}$ using a microplate spectrophotometer (BioTek Instruments, Inc., Winooski, VT, USA). The experiment was repeated three times.

Colony formation assay. LNCaP and DU-145 cells (200 cells/well) were seeded into 6-well plates and transfected with $50 \mathrm{nM}$ miR-301a-3p mimic, anti-miR-301a-3p or miR-NC at $37^{\circ} \mathrm{C}$ for $48 \mathrm{~h}$. Subsequently, the transfected cells were cultured at $37^{\circ} \mathrm{C}$ for 14 days in growth medium containing $0.3 \%$ noble agar to allow formation of natural colonies. The cells were stained with $0.1 \%$ crystal violet in $70 \%$ ethanol at room temperature for $20 \mathrm{~min}$. The number of stained colonies was counted under an inverted microscope. The experiment was repeated three times.

Cell invasion assay. Cell invasive ability was examined using a Transwell filter precoated with Matrigel (BD Bioscience, San Jose, CA, USA). Transfected cells ( $1 \times 10^{4}$ cells) in $200 \mu 1$ serum-free medium were added to the upper chamber, and $500 \mu \mathrm{l}$ of growth medium containing $10 \%$ FBS was added to the lower chamber as a chemoattractant. Cells were incubated for $24 \mathrm{~h}$ at $37^{\circ} \mathrm{C}$, and the invading cells on in the lower filter side were stained with $0.1 \%$ crystal violet for $20 \mathrm{~min}$ at room temperature and counted under an inverted fluorescence microscope (Olympus Corporation). The experiment was repeated three times.

Plasmid construction and dual-luciferase assay. The target reaction between miRNA and RUNX3 was predicted using targetscan (http://www.targetscan.org/vert_72/). Wild-type (wt) RUNX3 3'-UTR containing the predicted miR-301a-3p binding site was synthesized as described above using the following sequences: Forward 5'-CTAGTA TGGAGCTGGGTGGAAACTGCTTTGCACTATCGTTTG 
A

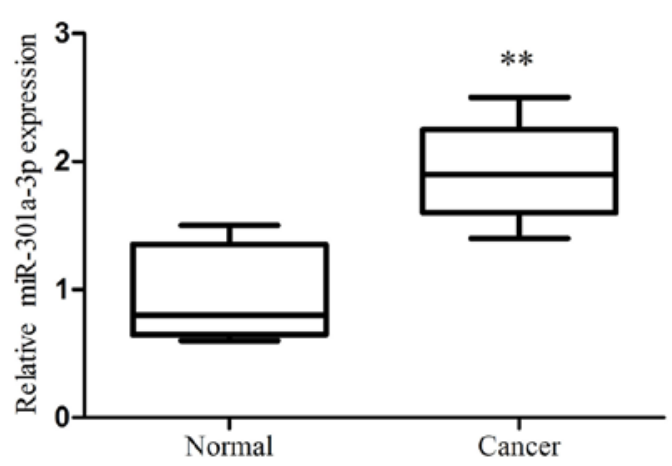

C

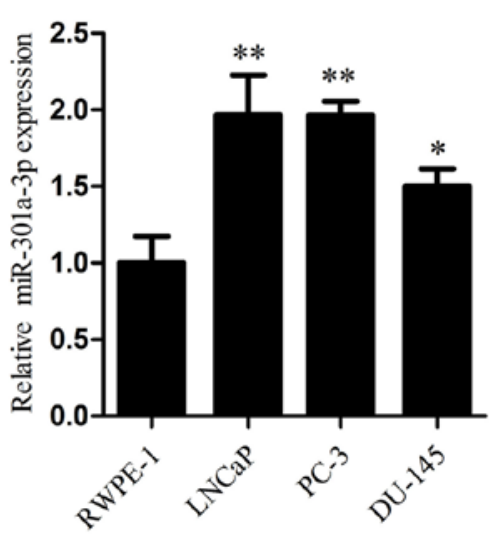

$\mathrm{B}$

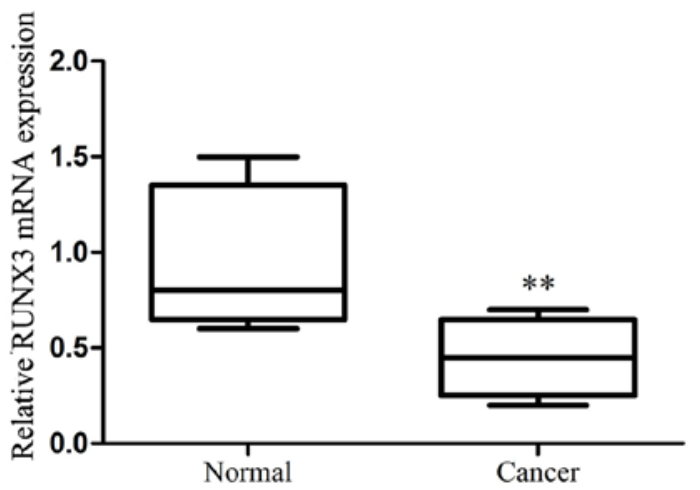

$\mathrm{D}$

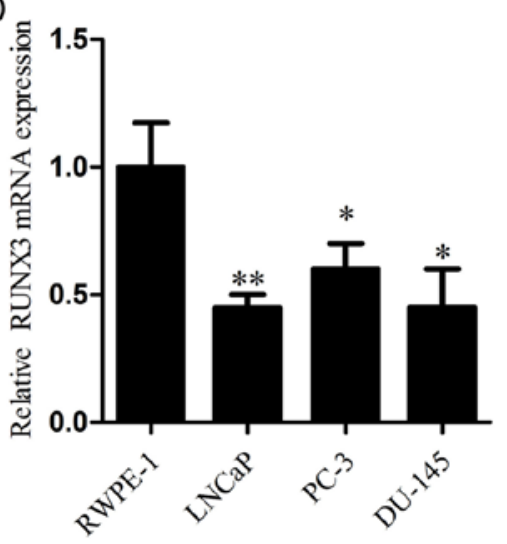

Figure 1. miR-301a-3p and RUNX3 mRNA expression levels in prostate cancer tissues and prostate cancer cell lines. (A) The expression of miR-301a-3p in 15 paired prostate cancer tissues and adjacent normal prostate tissues were measured by RT-qPCR. (B) The mRNA expression of RUNX3 in the prostate cancer and normal tissues was measured by RT-qPCR. ${ }^{* *} \mathrm{P}<0.01$ vs. Normal group. (C) The expression of miR-301a-3p in prostate cancer cell lines PC-3, LNCaP, DU-145, LAPC9, LAPC4 and the normal prostate epithelial cell line RWPE-1 were measured by RT-qPCR. (D) RUNX3 mRNA expression in the prostate cancer cell lines was measured by RT-qPCR. "P $<0.05$ and ${ }^{* *} \mathrm{P}<0.01$ vs. RWPE-1. miR, microRNA; RT-qPCR, reverse transcription-quantitative polymerase chain reaction.

CTTGGTGTTTGTTTTA3', reverse 5'-CGCGTAAAACAA ACACCAAGCAAACGATAGTGCAAAGCAGTTTCC ACCCAGCTCCATA-3'. The mutant mut) RUNX3 3'-UTR construct was designed to mutate three intermittent nucleotides that are complementary to the miR-301a-3p seed-region. The mutant primer sequences were: Forward 5'-CTAGTA TGGAGCTGGGTGGAAACTGCTTAGgAgTATCGTTTG CTTGGTGTTTGTTTTA-3', reverse 5'-CGCGTAAAACAA ACACCAAGCAAACGATACTCCTAAGCAGTTTCCACC CAGCTCCATA-3'. The strands were annealed and cloned into the pMIR-REPORT miRNA expression reporter vector (Applied Biosystems; Thermo Fisher Scientific, Inc.) using $B a m$ HI and EcoRI. 293T cells were co-transfected with either pMIR-RUNX3 wt or pMIR-RUNX3 mut plasmid containing firefly luciferase together with the $\mathrm{pRL}$-TK vector (Promega Corporation, Madison, WI, USA) containing Renilla luciferase and miR-301a-3p mimic or miR-NC using Lipofectamine 2000. Relative luciferase activity was calculated $36 \mathrm{~h}$ post-transfection by the Dual-Luciferase Reporter Assay (Promega Corporation), according to the manufacturer's protocol. The experiment was repeated three times. Luciferase expression was normalized to Renilla luciferase.

Western blot analysis. A total of $30 \mu \mathrm{g}$ protein was extracted from $6 \mathrm{~mm}$ tissue sample or $1 \times 10^{7}$ cell from LNCaP and DU-145 cancer cell line. The protein was separated by $8 \%$
SDS-PAGE, followed by electrophoretic transfer to nitrocellulose membranes for $1.5 \mathrm{~h}$ at $100 \mathrm{~V}$. Membranes were blocked with $5 \% \mathrm{BSA}$ for $1 \mathrm{~h}$ at room temperature and probed with primary antibodies (Cell Signaling Technology, Inc., Danvers, MA, USA), including mouse anti-human RUNX3 (cat. no. 13089; 1:1,000) and mouse anti-human $\beta$-actin (cat. no. 3700; 1:2,000) overnight at $4^{\circ} \mathrm{C}$. Membranes were washed with PBS and incubated with a horseradish peroxidase-conjugated anti-mouse secondary antibody (cat. no. 7076; 1:2,000; Cell Signaling Technology, Inc.) for $1 \mathrm{~h}$ at room temperature. Blots were developed using Enhanced Chemiluminescence kit (EMD Millipore, Billerica, MA, USA). Proteins were detected using a ChemiDoc XRS imaging system and Quantity One ${ }^{\circledR}$ 1-D analysis software (Bio-Rad Laboratories, Inc., Hercules, CA, USA). Relative protein expression was normalized to $\beta$-actin. The experiment was repeated three times.

Wht signaling activity assay. pcDNA3.0-RUNX3 overexpression vector were constructed using pcDNA3.0 plasmid (Invitrogen; Thermo Fisher Scientific, Inc.). The expression sequences encoding RUNX3 were amplified from cDAN, which was synthesized from prostate cancer cells, then sub-cloned into pcDNA3.0 plasmid. A total of $1 \times 10^{8} \mathrm{LNCaP}$ or DU-145 cancer cells were transfected with $10 \mathrm{ng}$ TOPFlash firefly luciferase reporter vector (Addgene, Inc., Cambridge, 
A

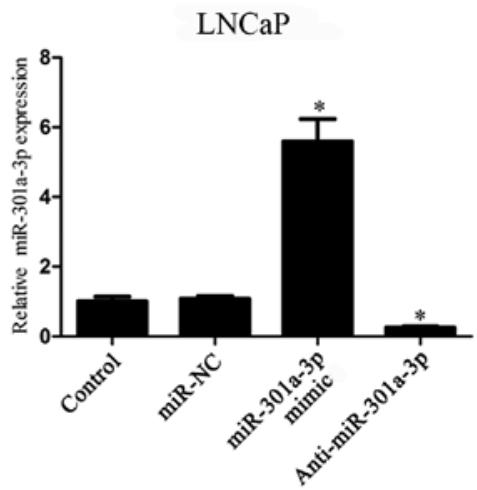

C

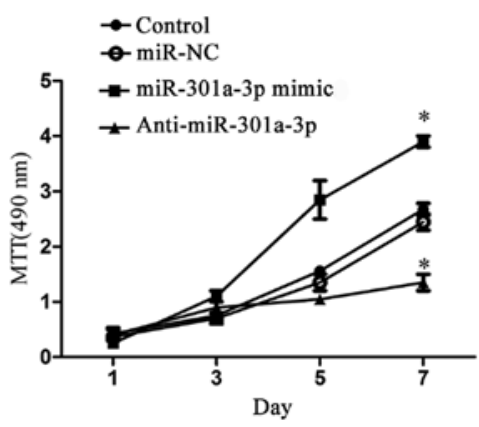

B

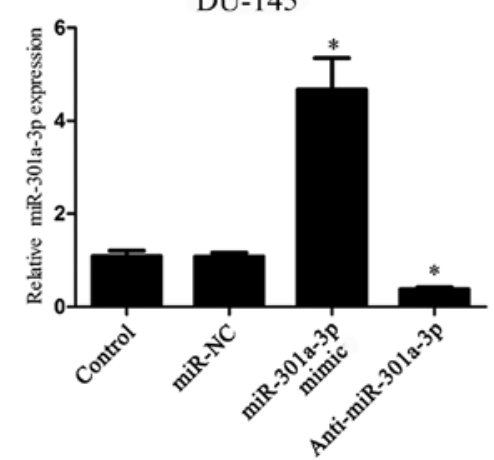

D

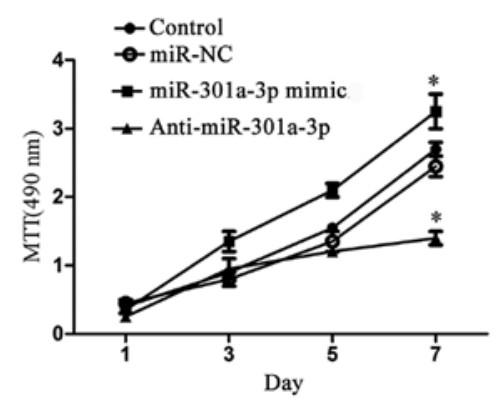

miR-301a-3p mimic
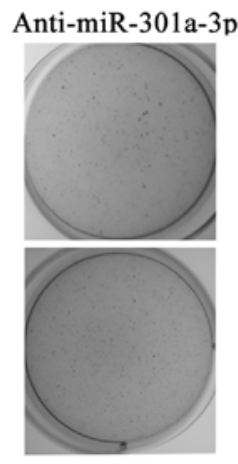

G

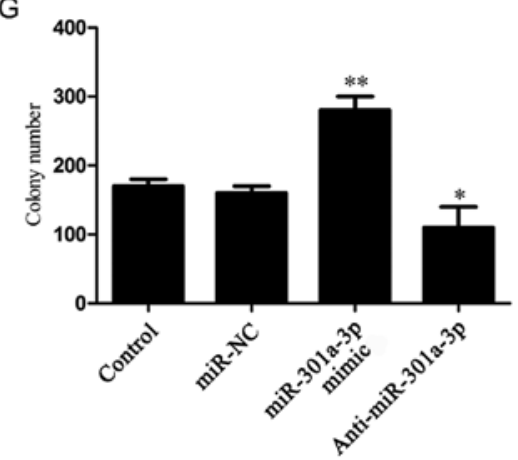

Figure 2. miR-301a-3p promotes proliferation and colony-forming capacity of prostate cancer cells. LNCaP and DU-145 prostate cancer cells were transfected with miR-NC, miR301a-39 mimics or anti-miR-301-3p; untransfected cells from each line were used as respective controls. (A and B) miR-301a-3p expression was measured by reverse transcription-quantitative polymerase chain reaction. (C and D) Cell proliferation was measured by MTT assay. (E) Colony-forming capacity of LNCaP and DU-145 ells was detected by colony formation assay. ( $\mathrm{F}$ and $\mathrm{G})$ Formed colonies were counted under a microscope. ${ }^{\mathrm{P}}<0.05$ and ${ }^{* *} \mathrm{P}<0.01$ vs. miR-NC. miR, microRNA; NC, negative control.

MA, USA) and 4 ng phRL-TK Renilla luciferase vectors (Promega Corporation) in the presence of $50 \mathrm{nM}$ miR-301a-3p mimics alone or combined with $50 \mathrm{nM}$ pcDNA3.0-RUNX3 overexpression vectors. Cells were incubated at $37^{\circ} \mathrm{C}$ for $48 \mathrm{~h}$, and the relative luciferase activity was determined using the Dual-Glo luciferase assay system (Promega Corporation).
Luciferase expression was normalized to Renilla luciferase. The experiment was repeated three times.

Statistical analysis. All numerical data were expressed as the mean \pm standard deviation. Statistical analyses were performed using the commercial statistical software SPSS version 11.5 
(SPSS Inc., Chicago, IL, USA). Differences between two groups were analyzed by paired two-tailed Student's t-test. Data of more than two groups were analyzed using one-way analysis of variance followed by a Bonferroni post hoc test. $\mathrm{P}<0.05$ was considered to indicate a statistically significant difference.

\section{Results}

miR-301a-3p expression is increased and RUNX3 mRNA expression is decreased in prostate cancer tissues and cell lines. To investigate the role of miR-301a-3p and RUNX3 in prostate cancer progression, miR-301a-3p and RUNX3 mRNA expression levels were determined in clinical prostate cancer tissues and paired adjacent normal tissues by RT-qPCR analysis. The results demonstrated that miR-301a-3p expression was significantly higher, whereas RUNX3 mRNA expression was significantly lower in prostate cancer tissues compared with the respective expression levels in paired adjacent normal tissues (Fig. 1A and B). In addition, the expression levels of miR-301a-3p and RUNX3 mRNA were examined in several prostate cancer cell lines, with the non-tumorigenic prostate epithelial cell line RWPE-1 used as the control. The data revealed that miR-301a-3p was significantly increased, whereas RUNX3 mRNA was significantly decreased in the prostate cancer cell lines compared with the respective expression levels in RWPE-1 cells (Fig. 1C and D). These results demonstrated that miR-301a-3p is upregulated and RUNX3 is downregulated in prostate cancer, and this may be involved in the pathogenesis of prostate cancer.

miR-30la-3p promotes the proliferative and colony-forming capacity of prostate cancer cells. To investigate the function of miR-301a-3p in prostate cancer, LNCaP and DU-145 prostate cancer cells transfected with miR-301a-3p mimics or anti-miR-301a-3p. LNCaP, an miR-301 highly expressing cell line and DU-145, in which miR-301 expression is relatively low was chosen out of the three prostate cancer cell line. These cell lines were chosen to examine effect the difference in expression had on the result. RT-qPCR results demonstrated that the expression of miR-301a-3p was significantly increased in miR-301a-3p mimics-treated cells and significantly decreased in anti-miR-301-3p-treated cells compared with untransfected Control and miR-NC-transfected groups (Fig. 2A and B); no significant differences were identified between the Control and miR-NC groups. MTT assay revealed that the overexpression of miR-301a-3p significantly promoted prostate cancer cell proliferation, whereas the inhibition of miR-301a-3p expression significantly reduced prostate cancer cell proliferation $(\mathrm{P}<0.05$; Fig. 2C and D). Furthermore, the colony-forming capacity of prostate cancer cells was significantly increased by miR-301a-3p overexpression, whereas suppression of miR-301a-3p exhibited the opposite effects (Fig. 2E-G). No significant differences in proliferation and colony formation were indicated between the Control and miR-NC groups. These results indicated that miR-301a-3p may serve an oncogenic role in prostate cancer.

miR-301a-3p promotes the invasion of prostate cancer cells. To further investigate the functions of miR-301a-3p in prostate
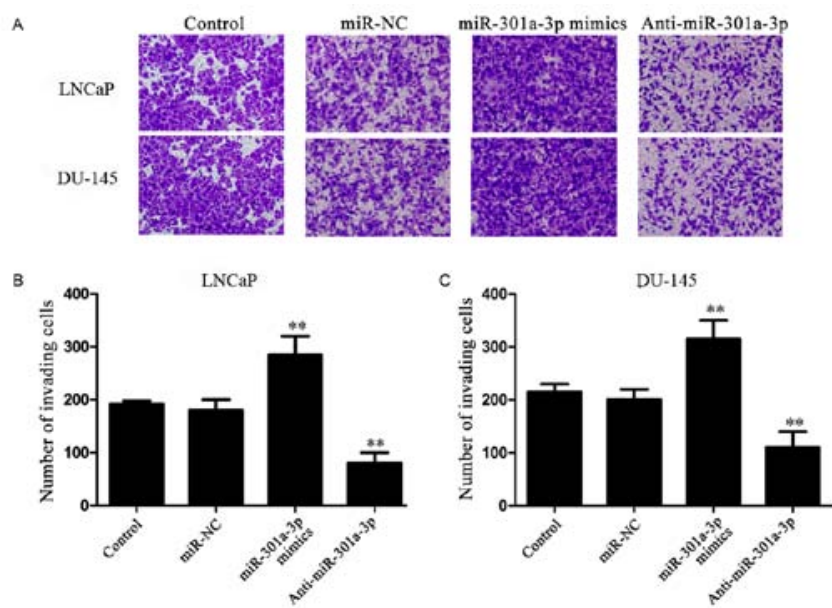

Figure 3. miR-301a-3p promotes the invasion and migration of prostate cancer cells. (A) Representative fields of LNCaP and DU-145 cells in the invasion assays. (B) Average number of invading LNCaP cells per field from three independent experiments. (C) Average number of invading DU+145 cells per field from three independent experiments. ${ }^{* *} \mathrm{P}<0.01$ vs. miR-NC.

cancer, cell migration (Fig. 3A and B) was performed on the transfected cells. LNCaP and DU-145 cells transfected with miR-301a-3p mimics exhibited a significantly increased number of invading cells compared with untransfected or miR-NC transfected cells. Conversely, inhibition of miR-301a-3p expression significantly reduced cell invasion. No significant differences in the numbers of invading cells were identified between the Control cells and the miR-NC-transfected cells.

miR-301a-3p targets RUNX3 in prostate cancer cells. To investigate the underlying mechanism by which miR-301a-3p controls the pathogenesis of prostate cancer, bioinformatics analysis was performed to predict target genes of miR-301a-3p. Of the predicted target genes (all illustrated in Fig. 4A) the tumor suppressor RUNX3 was selected for further analysis. RUNX3 was chosen because it is a tumor-related gene, and its function has been confirmed in various cancers.

To verify that RUNX3 is a target of miR-301a-3p, dual-luciferase reporter assays were performed; LNCaP and DU-145 cells were co-transfected with either wt or mut RUNX3 3'-UTR and either miR-301a-3p mimics or miR-NC. Luciferase activity in cells co-transfected with miR-301a-3p mimics and wt RUNX3 3'UTR was significantly reduced compared with cells resulted into a significant decrease in luciferase activity (Fig. 4B and C).

The effects of miR-301a-3p on RUNX3 expression were investigated in vitro. The data demonstrated that RUNX3 protein expression levels were significantly decreased in LNCaP and DU-145 cells transfected with miR-301a-3p mimics compared with untransfected Control cells (Fig. 4D and E). To further investigate the role of RUNX3, rescue experiments were conducted by co-transfecting cells with miR-301a-3p mimics and RUNX3 overexpression vector, which exhibited a significant increase in RUNX3 protein expression compared with cells transfected with miR-301a-3p mimics alone (Fig. 4D and E). Taken together, these results suggested that miR-301a-3p directly targets the 3'-UTR of RUNX3 and regulates RUNX3 expression in prostate cancer cells. 


\section{A 3' cgaAACUGUUAUGAUACGUGAC 5' hsa-miR-301a-3p 2474:5' gggUGGAAACUGCU - UỤGC̣AC̣Ua 3' RUNX3}

B

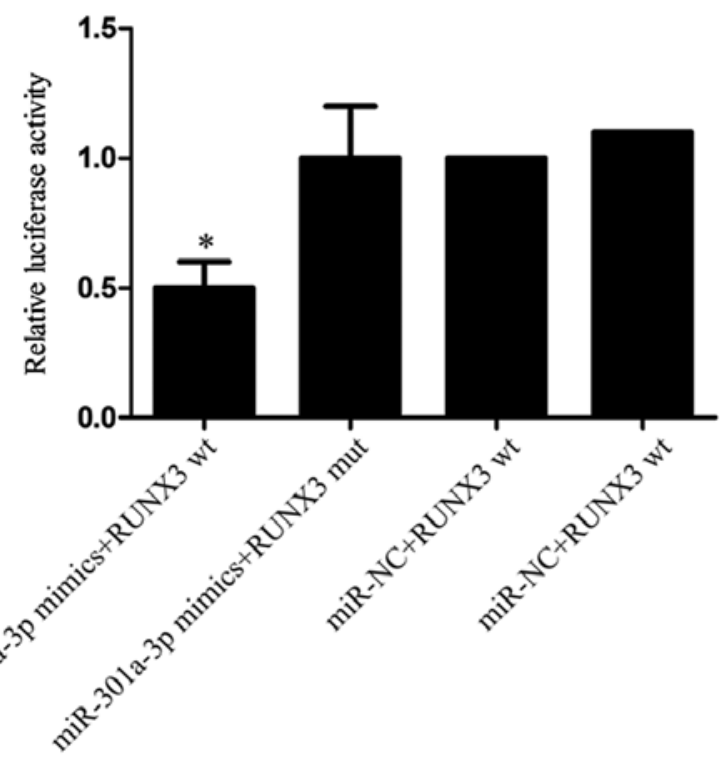

D
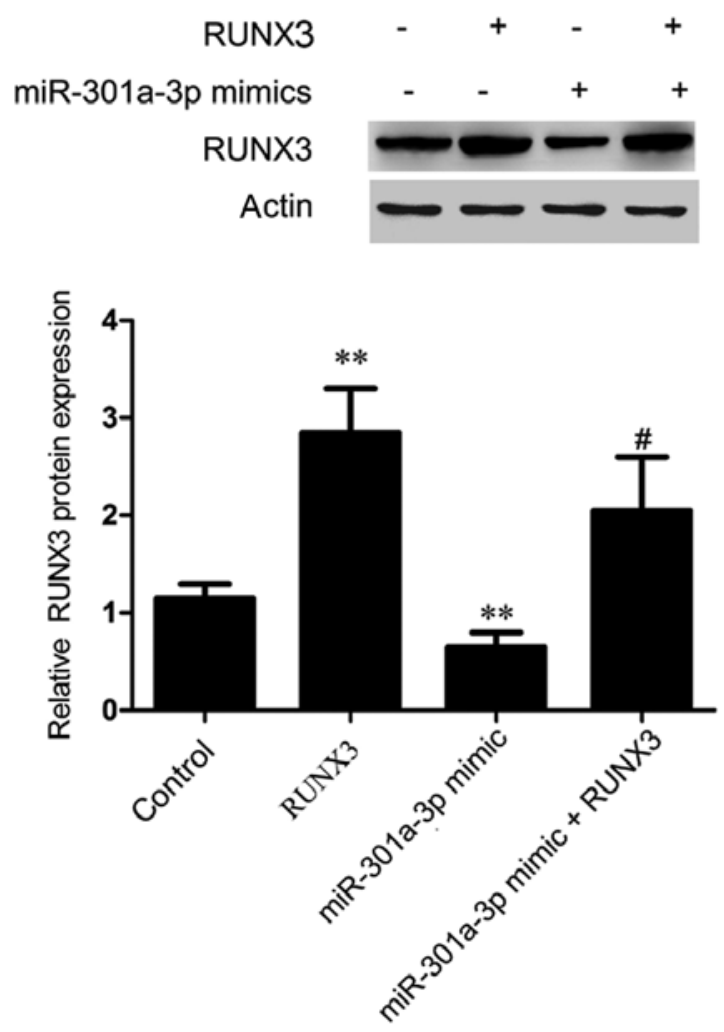

LNCaP

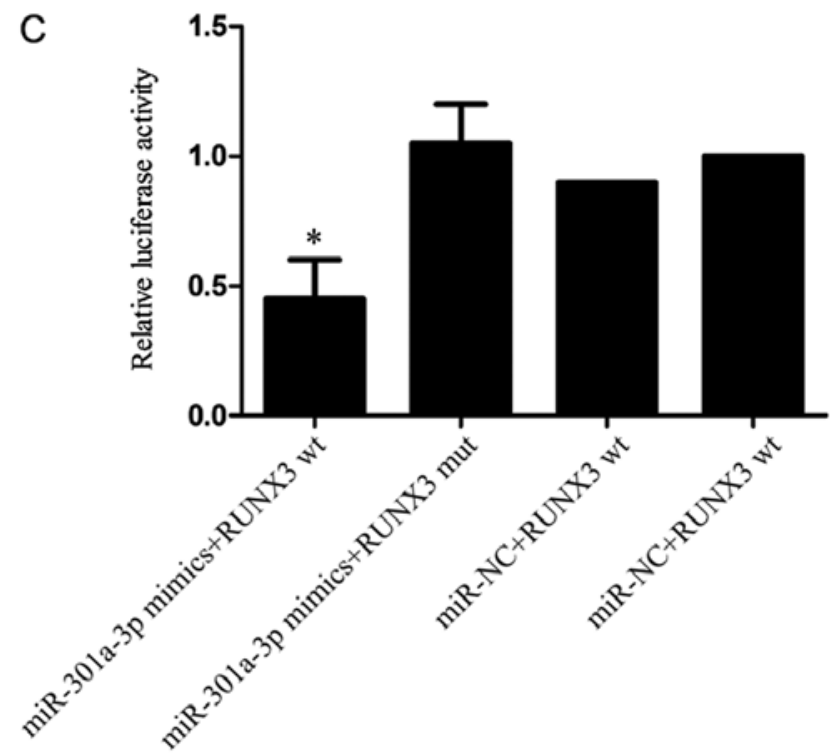

E

RUNX3

miR-301a-3p mimics

RUNX3

Actin

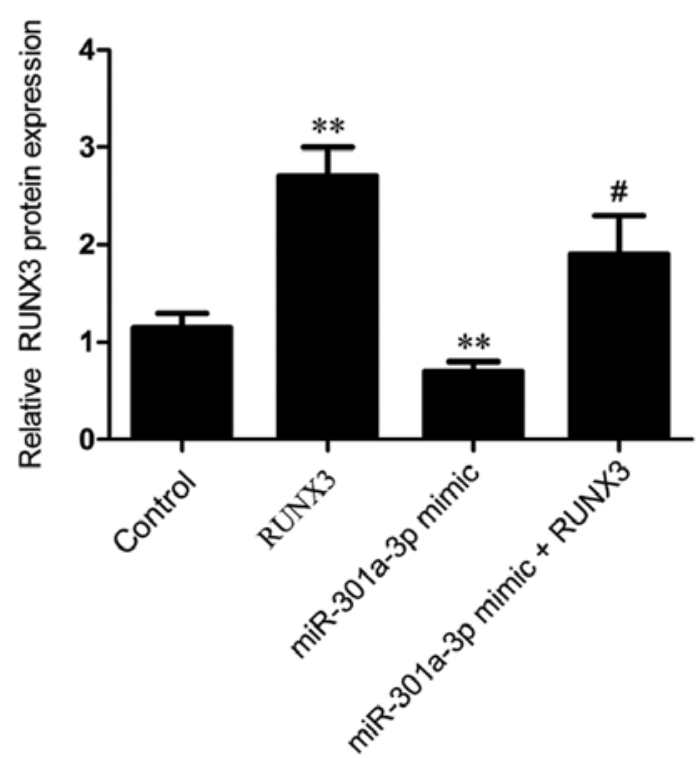

DU-145

Figure 4. miR-301a-3p targets RUNX3 3'-UTR in prostate cancer cells. (A) Putative binding site of miR-301a-3p in the 3'-UTR region of RUNX3 was identified using Target Scan. The mutated nucleotides are indicated using arrows. (B and C) Dual-luciferase reporter assays of (B) LNCaP and (C) DU-145 cells co-transfected with miR-301a-3p mimics or miR-NC and WT or MUT RUNX3 3'-UTR. * $\mathrm{P}<0.05$ vs. miR-NC + RUNX3 WT. (D and E) Protein expression levels of RUNX3 were measured by western blotting in (D) LNCaP and (E) DU-145 cells transfected with miR-301a-3p mimics, RUNX3 overexpression vector or a combination of the two; untransfected cells were used as a control. ${ }^{* *} \mathrm{P}<0.01$ vs. the Control group; ${ }^{*} \mathrm{P}<0.05$ vs. miR-301a-3p mimics group. miR, microRNA; MUT, mutant; NC, negative control; RUNX3, runt-related transcription factor 3; UTR, untranslated region; WT, wild-type.

miR-301a-3p regulates Wnt signaling pathway. To further elucidate the molecular role of miR-301a-3p in regulating prostate cancer, the effects of miR-301a-3p on the Wnt signaling pathway were examined by using a luciferase assay. The Wnt pathway was chosen for investigation as RUNX3 was proved to modulate the Wnt pathway in a number of 
A

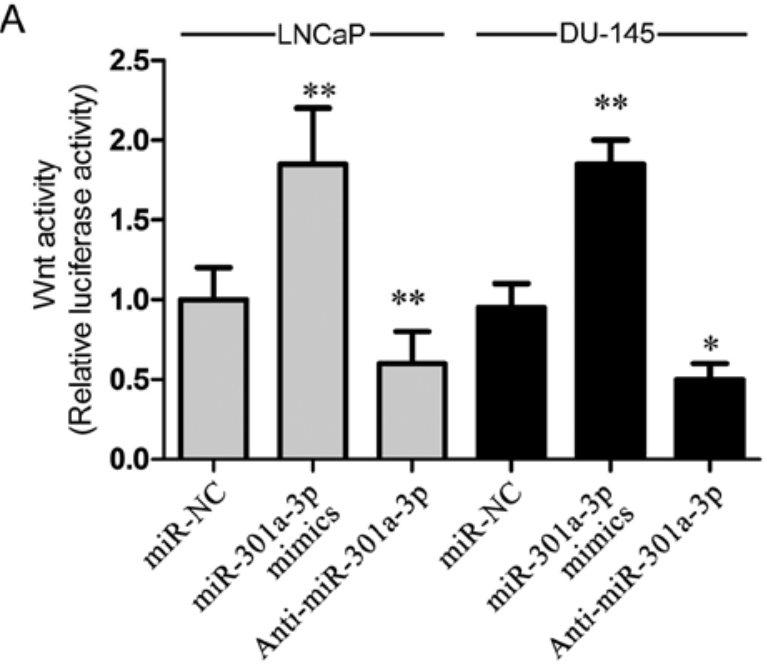

C

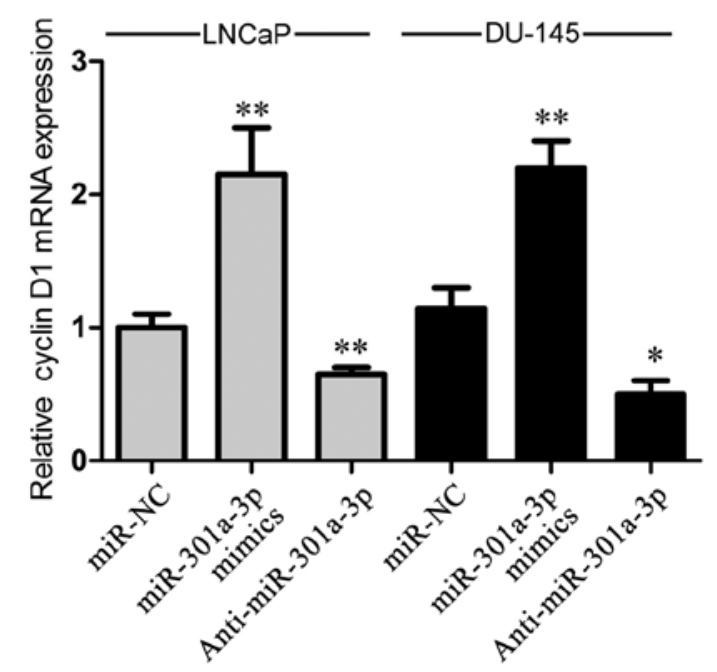

B

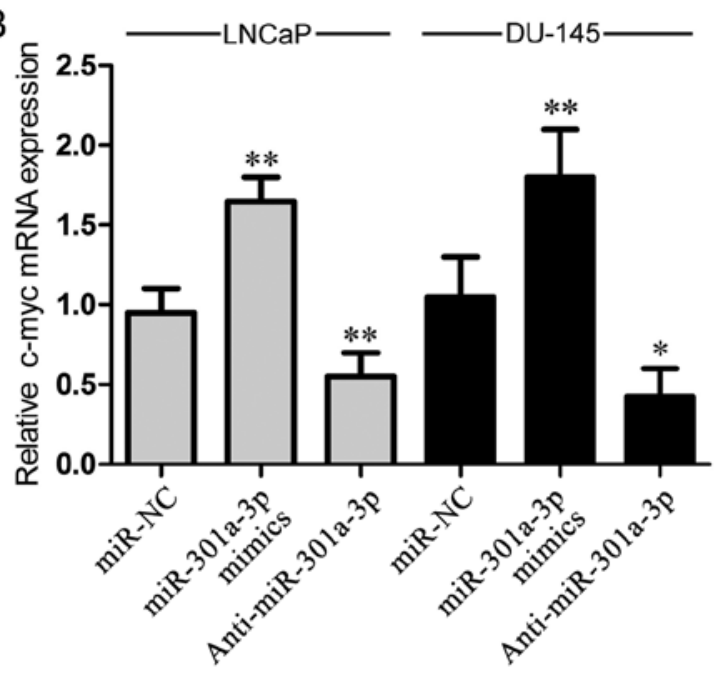

D

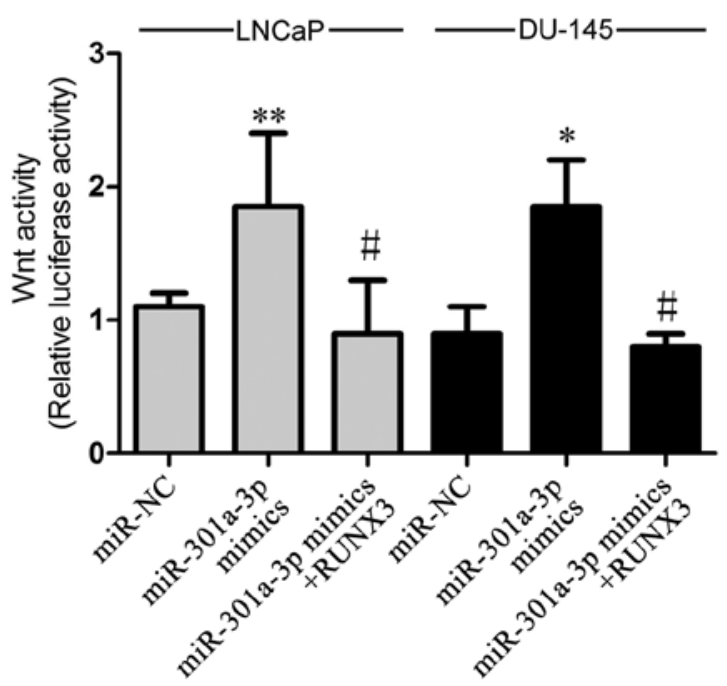

Figure 5. miR-301a-3p regulates Wnt signaling pathway. (A-C) LNCaP and DU-145 cells were transfected with miR-301a-3p mimics or anti-miR-301a-3p. (A) The effects of miR-301a-3p on the Wnt signaling pathway was determined by a Tcf-dependent TOPFlash reporter Wnt activity assay. (B) c-myc and (C) cyclin D1 mRNA expression levels in treated LNCaP and DU-145 cells were examined by reverse transcription-quantitative polymerase chain reaction. (D) Effects of RUNX3 overexpression on the Wnt signaling pathway was detected by Tcf-dependent TOPFlash reporter activity assays. ${ }^{*}<0.05$ and ${ }^{* *} \mathrm{P}<0.01$ vs. miR-NC; ${ }^{\text {P }}<0.05$ vs. miR-301a-3p mimics group. miR, microRNA; NC, negative control; Tcf, T-cell factor.

types of cancer and studies of the Wnt pathway are more prevalent. The data revealed that miR-301a-3p overexpression significantly increased Wnt activity, whereas miR-301a-3p inhibition reduced Wnt activity in LNCaP and DU-145 cells (Fig. 5A). To verify these results, the mRNA expression levels of downstream Wnt signaling genes c-myc and cyclin D1 were examined; the expression levels of c-myc and cyclin D1 were significantly increased by miR-301a-3p overexpression in LNCaP and DU-145 cells (Fig. 5B and C), whereas miR-301a-3p inhibition decreased the expression of c-myc and cyclin D1. These results indicated that miR-301a-3p may serve a role in regulating the Wnt signaling pathway. To investigate whether miR-301a-3p regulates Wnt pathway through RUNX3, a rescue assay was conducted by overexpressing RUNX3 in miR-301a-3p mimics-transfected cells. The data indicated that the promotional effects of miR-301a-3p on the Wnt signaling pathway was significantly reversed by RUNX3 overexpression (Fig. 5D).

\section{Discussion}

Prostate cancer is the second leading cause of cancer-related mortality in men worldwide (13). Although hormone therapy may initially be effective, the tumor may still enter the insensitive period and metastasize (14). Previous studies suggested that miRNAs may serve important roles in regulating the progression of prostate cancer $(15,16)$; therefore, it is of great importance to identify prostate cancer-associated miRNAs that may be used as biomarkers for prostate cancer diagnosis and treatment. Results from the present study revealed that miR-301a-3p expression was significantly increased in prostate cancer tissues and cell lines. In addition, functional analysis indicated that miR-301a-3p may serve a role in regulating prostate cancer cell proliferation and invasion and may serve an important role in prostate cancer progression.

Previous studies have reported that miR-301a-3p is highly expressed in various types of human cancers, 
including pancreatic tumors, hepatocellular carcinoma, breast cancer and small cell lung cancer (17). Results from the present study demonstrated that miR-301a may function as a novel oncogene in prostate cancer and contribute to tumor progression of prostate cancer. The biological role of miRNAs is dependent on its target genes. The present study demonstrated that miR-301a-3p directly targets the 3'-UTR of RUNX3 and regulates RUNX3 expression, which was consistent with a former study (7). RUNX3 expression was previously reported to be reduced in many types of human cancers, including melanoma, renal cell carcinoma, colorectal cancer and breast cancer $(18,19)$. RUNX3 was also reported to regulate migration and proliferation of human colorectal cancer (20). The present study results demonstrated that RUNX3 is downregulated in prostate cancer. A previous study reported that targeted restoration of RUNX3 expression in hepatocellular cancer cells inhibits tumor development (21). Another study suggested that RUNX3 controls the differentiation and growth of gastric cancer cells (22). The present study suggested that miR-301a-3p-induced invasion and proliferation may depend on the direct post-transcriptional downregulation of RUNX3 expression in prostate cancer.

Aberrant activation of the Wnt signaling pathway is frequently associated with prostate cancer (23). Given the regulatory effects of RUNX3 on the Wnt signaling pathway (24), it was hypothesized that miR-301a-3p may also influence the Wnt signaling pathway. The present study results indicated that miR-301a-3p may promote the Wnt signaling pathway by affecting RUNX3 expression.

In conclusion, the present study demonstrated that miR-301a-3p was upregulated and affected the proliferation and invasive ability of prostate cancer cells by regulating of RUNX3 expression and Wnt signaling. Therefore, miR-301a-3p may act as an oncogene through targeting RUNX3 in prostate cancer. These results may aid in the further elucidation of potential molecular mechanisms of prostate cancer development, and may have therapeutic value in the future.

\section{Acknowledgements}

Not applicable.

\section{Funding}

No funding was received.

\section{Availability of data and materials}

The datasets used and/or analyzed during the current study are available from the corresponding author on reasonable request.

\section{Authors' contributions}

LF designed the present study and wrote the manuscript. YW and WH performed the experiments. WHW participated in design of the present study and helped revise the manuscript.

\section{Ethics approval and consent to participate}

Written informed consent was obtained from the participating patients prior to the start of the study, and the study was approved by The Institutional Human Experiment and Ethics Committee of China-Japan Union Hospital of Jilin University.

\section{Patient consent for publication}

Written informed consent was obtained from the participating patients prior to the start of the study.

\section{Competing interests}

The authors declare they have no competing interests.

\section{References}

1. Prorok PC, Wright P, Riley TR, Kramer BS, Berg CD and Gohagan JK: Overall and multiphasic findings of the prostate, lung, colorectal and ovarian (PLCO) randomized cancer screening trial. Rev Recent Clin Trials: Apr 9, 2018 (Epub ahead of print).

2. Devlin HL and Mudryj M: Progression of prostate cancer: Multiple pathways to androgen independence. Cancer Lett 274: 177-186, 2009.

3. Winter J, Jung S, Keller S, Gregory RI and Diederichs S: Many roads to maturity: microRNA biogenesis pathways and their regulation. Nat Cell Biol 11: 228-234, 2009.

4. Manikandan J, Aarthi JJ, Kumar SD and Pushparaj PN: Oncomirs: The potential role of non-coding microRNAs in understanding cancer. Bioinformation 2: 330-334, 2008.

5. Wang YL, Wu S, Jiang B, Yin FF, Zheng SS and Hou SC: Role of MicroRNAs in prostate cancer pathogenesis. Clin Genitourin Cancer 13: 261-270, 2015.

6. Aakula A, Kohonen P, Leivonen SK, Mäkelä R, Hintsanen P, Mpindi JP, Martens-Uzunova E, Aittokallio T, Jenster G, Perälä M, et al: Systematic identification of MicroRNAs that impact on proliferation of prostate cancer cells and display changed expression in tumor tissue. Eur Urol 69: 1120-1128, 2016.

7. Wang M, Li C, Yu B, Su L, Li J, Ju J, Yu Y, Gu Q, Zhu Z and Liu B: Overexpressed miR-301a promotes cell proliferation and invasion by targeting RUNX3 in gastric cancer. J Gastroenterol 48: 1023-1033, 2013.

8. Xu N, Shen C, Luo Y, Xia L, Xue F, Xia Q and Zhang J: Upregulated miR-130a increases drug resistance by regulating RUNX3 and Wnt signaling in cisplatin-treated HCC cell. Biochem Biophys Res Commun 425: 468-472, 2012.

9. Zhang Y, Lu Q and Cai X: MicroRNA-106a induces multidrug resistance in gastric cancer by targeting RUNX3. FEBS Lett 587: 3069-3075, 2013.

10. Zheng Y, Wang R, Song HZ, Pan BZ, Zhang YW and Chen LB: Epigenetic downregulation of RUNX3 by DNA methylation induces docetaxel chemoresistance in human lung adenocarcinoma cells by activation of the AKT pathway. Int J Biochem Cell Biol 45: 2369-2378, 2013.

11. Miko E, Czimmerer Z, Csanky E, Csánky E, Boros G, Buslig J, Dezso B and Scholtz B: Differentially expressed microRNAs in small cell lung cancer. Exp Lung Res 35: 646-664, 2009.

12. Livak KJ and Schmittgen TD: Analysis of relative gene expression data using real-time quantitative PCR and the 2(-Delta Delta C(T)) method. Methods 25: 402-408, 2001.

13. Torre LA, Bray F, Siegel RL, Ferlay J, Lortet-Tieulent J and Jemal A: Global cancer statistics, 2012. CA Cancer J Clin 65: 87-108, 2015.

14. Nakabayashi M, Hayes J, Taplin ME, Lefebvre P, Lafeuille MH, Pomerantz M, Sweeney C, Duh MS and Kantoff PW: Clinical predictors of survival in men with castration-resistant prostate cancer: Evidence that Gleason score 6 cancer can evolve to lethal disease. Cancer 119: 2990-2998, 2013.

15. Yao J, Xu C, Fang Z, Li Y, Liu H, Wang Y, Xu C and Sun Y: Androgen receptor regulated microRNA miR-182-5p promotes prostate cancer progression by targeting the ARRDC3/ITGB4 pathway. Biochem Biophys Res Commun 474: 213-219, 2016. 
16. Zhang H, Li S, Yang X, Qiao B, Zhang Z and Xu Y: miR-539 inhibits prostate cancer progression by directly targeting SPAG5. J Exp Clin Cancer Res 35: 60, 2016.

17. Shi W, Gerster K, Alajez NM, Tsang J, Waldron L, Pintilie M, Hui AB, Sykes J, P'ng C, Miller N, et al: MicroRNA-301 mediates proliferation and invasion in human breast cancer. Cancer Res 71: 2926-2937, 2011.

18. Zhang Z, Chen G, Cheng Y, Martinka M and Li G: Prognostic significance of RUNX3 expression in human melanoma. Cancer 117: 2719-2727, 2011.

19. Chen F, Bai J, Li W, Mei P, Liu H, Li L, Pan Z, Wu Y and Zheng J: RUNX3 suppresses migration, invasion and angiogenesis of human renal cell carcinoma. PLoS One 8: e56241, 2013.

20. Lee CW, Ito K and Ito Y: Role of RUNX3 in bone morphogenetic protein signaling in colorectal cancer. Cancer Res 70: 4243-4252, 2010.
21. Chen Y, Wang X, Cheng J, Wang Z, Jiang T, Hou N, Liu N, Song $T$ and Huang C: MicroRNA-20a-5p targets RUNX3 to regulate proliferation and migration of human hepatocellular cancer cells. Oncol Rep 36: 3379-3386, 2016.

22. Wei D, Gong W, Oh SC, Li Q, Kim WD, Wang L, Le X, Yao J, Wu TT, Huang S and Xie K: Loss of RUNX3 expression significantly affects the clinical outcome of gastric cancer patients and its restoration causes drastic suppression of tumor growth and metastasis. Cancer Res 65: 4809-4816, 2005.

23. Fukamachi H, Ito K and Ito Y: Runx3-/-gastric epithelial cells differentiate into intestinal type cells. Biochem Biophys Res Commun 321: 58-64, 2004.

24. Ju X, Ishikawa TO, Naka K, Ito K, Ito Y and Oshima M: Context-dependent activation of Wnt signaling by tumor suppressor RUNX3 in gastric cancer cells. Cancer Sci 105: 418-424, 2014. 